\title{
N $89-23548$
}

The Space Particle Environment

A. L. Vampola
The Aerospace Corporation, P.O. Box 92957, Los Angeles, CA 90009 


\section{ABSTRACT}

This paper is a tutorial covering the energetic charged particle environment in the Earth's magnetosphere. It provides an overview of trapped particle morphology, the geometry of the trapping regions, the radiation environmental models, the current status of these models, and future modelling requirements.

\section{INTRODUCTION}

The extensive use of space for platforms for communications, surveillance such as weather andEarth resources, science research, military objectives, and manned activities results in a critical requirement for knowledge about the energetic particle environment in space. Radiation damage to circuits and materials, background effects in sensors, hazard to personnel, spurious effects in circuits: all are the result of this energetic particle environment. Utilization of space is continuing to increase. With this increase comes an equivalent increase in the number of personnel who have to have basic knowledge about and access to information about the space particle environment. The material here is intended to provide an overview in the areas of the dynamics of the particle environment, trapped radiation morphology, current trapped radiation models, and future modelling activity in this field. The references accompanying this discussion can serve as a convenient source for more detailed information in this field.

A Geiger-Muller tube launched February 1, 1958 on Explorer I by Dr. James Van Allen is considered to have produced the discovery of the trapped radiation belts surrounding the earth, but such a phenomenon had been predicted prior to the launch of the first artificialEarth satellite. A great flurry of activity aimed at understanding the radiation belts (sometimes called the Van Allen belts) ensued. However the myriad of measurements initially resulted in the acquisition of data rather than the acquisition of understanding. Part of the problem was the fact that data were being organized in terms of the orbital parameters longitude-latitude-altitude, a three parameter space, and the quantity of data was insufficient to provide a sufficient density of data points in any part of space to produce a reliable picture of the particle population. In addition to the three spatial parameters, there were also energy, time, and species to contend with. Some instruments made integral energy measurements (detected everything above a particular energy threshold, such as Geiger counters which counted any charged particle which could penetrate its window or walls), some made differential energy measurements, some made unidirectional measurements using collimators, others accepted particles coming from anywhere within a $2 \pi$ or $4 \pi$ steradian angle. Some detectors were on spinning vehicles, other on stabilized platforms. Since the particle population is not isotropic (same intensity in all directions), further confusion was possible. The first major step toward producing order out of chaos was taken by Prof. Carl Mcllwain when he introduced a new variable, L, based on the second adiabatic invariant of particle motion [1], which with the magnetic field coordinate $B$ formed a two-parameter space to replace the three spatial coordinates used to describe satellite orbits. The variable $\mathrm{L}$ is discussed in the next section.

In 1965, NASA funded Dr. James Vette of the Aerospace Corporation to produce model electron and proton environments using the data then available from the various satellites. All the particle data sets available within the US were assembled and incorporated into model environments--AE1 (Aerospace Electron model environment number 1 ) and AP1 (Aerospace Proton model environment number $\underline{1}$ ) were the result. These initial models could not be considered to be more than educated guesses. Great effort was expended in translating the various data sets into a common parameter space. (As noted above, different variables controlled the various experimental measurements of the trapped particles.) Since with 
sparse data sets differential energy measurements could be converted reliably into integral fluxes and unidirectional measurements into omnidirectional but not vice-versa, the "lowest common denominator" ap)proach was used: fluxes were specified as integral-omnidirectional fluxes as a function of location in $B, L$ space. This is still the mode used for most trapped particle environment models. Two to three orders of magnitude disagreement between ostensibly similar measurements were the norm rather than the exception, even in some cases where the measurements were made at the same time by different instruments on the same satellite. It was realized that producing a reliable environmental model was going to be a gargantuan task. The National Space Science Data Center was set up by NASA at the Goddard Space Flight Center with Dr. Vette as its director and a major effort at space environment modelling was begun, one that continues at a low level to this day. The models produced by those efforts will be discussed later in this presentation.

\section{TRAPPED PARTICLE MORPHOLOGY}

In this section, three subjects will be discussed: trapped particle motions, McIlwain's "L" parameter, and the general configuration of the radiation belts. The discussion is at an introductory level: no previous knowledge about the particle radiation environment is assumed. The intent is to provide sufficient background material about radiation belt morphology that the rest of this tutorial and the other presentations in these proceedings which deal with particle radiation effects will be understood in context.

\section{Trapped Particle Motions}

An understanding of the dynamics of the radiation belts requires some knowledge of the dynamics of an individual particle. The three basic particle motions in the Earth's magnetosphere with which we are concerned are gyration or cyclotron motion, bounce, and drift. The gyration is about the local magnetic field line, the bounce motion is from one end of the field line to the other (one hemisphere to the other), and the drift is around the world in longitude. These motions are a consequence of the behavior of a charged particle with forces acting on it moving in a non-uniform magnetic field.

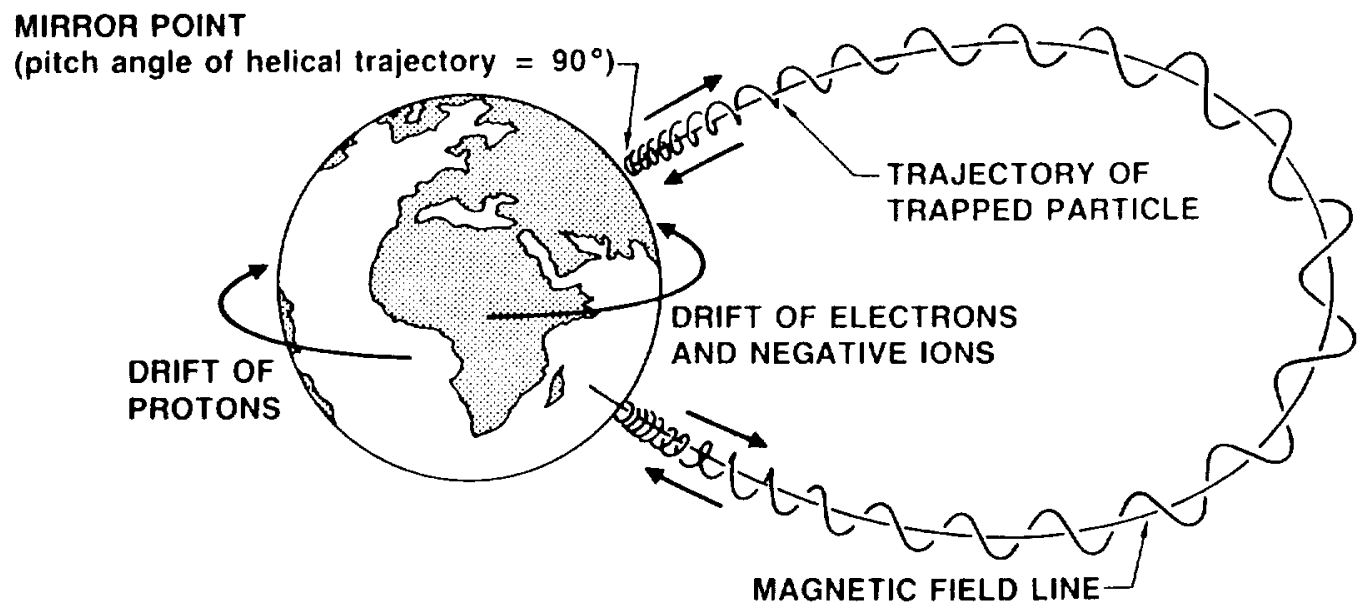

Figure 1. Geomagnetically Trapped Particle Motions

In a uniform magnetic field $\mathbf{B}$, a charged particle $q$ moving with velocity $\mathbf{v}$ experiences a force which appears as an electric field $\mathbf{E}$ at right angles to both the direction of the field $\mathbf{B}$ and the component of the velocity vector perpendicular to that field $\left(E=-q \mathbf{v}_{\mathbf{x}} \mathbf{B}\right.$ in vector notation). Since at each instant this electric field tends to change the direction of the particle, which in turn changes the direction of the effective electric field, the particle executes a circular path---it gyrates about the field. This coupling of forces also results in a complementary behavior: a charged particle initially at rest in a magnetic field which has a force imposed upon it will move in a net direction perpendicular to both the force and the magnetic field 
(components of force along the field are ignored here). The three basic motions of trapped particles in the magnetic field are a consequence of these force couplings and of the fact that the geomagnetic field has curvature and intensity gradients. Because of the curvature and intensity gradients, the particle's gyration path does not close upon itself, resulting in a drift motion. The motions are shown in Figure 1.

The direction of gyration follows the "right hand rule" for both electrons and ions; since the charges are opposite for the two types of particles, the direction of gyration is opposite for the electrons and ions, and therefore the direction of drift is also opposite, with electrons and negative ions drifting eastward and protons and other positive ions drifting westward. The frequency of gyration, called the Larmor frequency, is given by

$$
\begin{aligned}
& \mathrm{f}_{L}=\Omega_{1} / 2 \pi=-\mathrm{q} B / 2 \pi \mathrm{m}_{0} \gamma \mathrm{c} \\
& \text { where: } \begin{array}{l}
\mathrm{q}=\text { charge on the electron or ion } \\
\mathrm{B}=\text { local magnetic field } \\
\mathrm{m}_{0}=\text { rest mass of the electron or ion } \\
\gamma=\text { relativistic mass ratio of the particle, } 1 /\left(1-\mathrm{v}^{2} / \mathrm{c}^{2}\right) 1 / 2 \\
v=\text { velocity of the particle } \\
\mathrm{c}=\text { velocity of light }
\end{array}
\end{aligned}
$$

Note that since the frequency of gyration is proportional to $\mathrm{B}$, it is not constant along the field line. It is a minimum at the magnetic equator and increases as the particle moves away from the equator. Typical equatorial frequencies at $1000 \mathrm{~km}$ altitude are around $0.5 \mathrm{MHz}$ for very low energy electrons and about $300 \mathrm{~Hz}$ for low energy protons. High energy particles have lower gyrofrequencies because of their greater (relativistic) mass.

\section{The First Adiabatic Invariant}

If one analyzes the path of a particle gyrating in a magnetic field, one observes that it encloses a fixed amount of flux which depends on the momentum of the particle perpendicular to the magnetic field. Under static conditions, the flux can't "leak" out of the path. This flux quantity, which is the origin of the magnetic moment of the particle, is invariant as long as conditions are adiabatic; i.e., the magnetic field is quiescent and no energy is added to the particle. It is called the first adiabatic invariant. The radius of gyration is inversely proportional to $\mathrm{B}$. Thus the total flux enclosed by the path (flux density times area) is inversely proportional to the square of the magnetic field. But, if the magnetic field increases, thereby decreasing the radius of gyration, the perpendicular momentum of the particle has to increase to conserve the magnetic moment--to keep any of the flux from leaking out of the enclosed path. Otherwise the flux area enclosed by the gyration path would have decreased by $\mathrm{B}^{2}$ while the flux density increased by only $\mathrm{B}$. This has two interesting consequences:

First, it produces the bounce motion of particles in the geomagnetic field. A particle starting out at the equator with a component of velocity along the field line will travel a helical path to lower altitude with the field line as a guiding center. As it moves, it is moving in an increasing field. In order to maintain a constant magnetic moment, the momentum component perpendicular to the magnetic field has to increase (in the absence of such an increase, the magnetic moment would be decreasing as $1 / \mathrm{B}$ ). The only sources of energy to provide this perpendicular momentum increase are the magnetic field and the particle velocity. In a quiescent field, all of the momentum increase is obtained from the particle motion by converting momentum parallel to the field into momentum perpendicular to the field. When this source is exhausted, the particle motion parallel to the field line is zero and the particle is gyrating at a field intensity $B_{m}$, called the mirror $B$. The gradient in the field then reverses the process (called mirroring) and the particle travels a helical path to the other hemisphere where it again mirrors at a magnetic field intensity $B_{m}$ exactly equal to the previous one. The two mirror points are called conjugate points because they are joined by the field line which is the guiding center of this helical motion. The particle actually spends most of its time at these mirror points.

The second interesting consequence which follows from conservation of the first adiabatic invariant is acceleration of the particle by an increasing magnetic field. As noted above, the only sources of energy to 
maintain a constant magnetic moment in an increasing field are the particle motion and the field. If the field is changing, the particle may maintain its magnetic moment by increasing its perpendicular momentum at the expense of the magnetic field. Consider a particle that is mirroring so that all of its momentum is perpendicular momentum. If the field then increases, the field must accelerate the particle to increase the particle's perpendicular momentum (which must be done to conserve the total flux enclosed within the particle's gyration path). Note that the particle still has the same magnetic moment after it has been accelerated as it had previously, but it is now at a higher energy. The reverse process also works: a decreasing field will decelerate particles. Geomagnetic activity does both, producing radial displacements in the process. If the third adiabatic invariant, discussed below, is violated, the result is radial diffusion (a process in which particles initially on the same field line are transported to higher and lower field lines) which results in a net increase of energetic particles in the outer zone. These energetic particles then diffuse both inward and outward.

\section{The Second Adiabatic Invariant}

The bounce motion described above also has an associated invariant, called the second adiabatic invariant. Basically it is the total magnetic field energy contained within the envelope of the helical path between mirror points. (This function, like the other invariants, can be evaluated as a line integral. This will be discussed in more detail under McIlwain's L Parameter.) Note that if the magnetic field is increased, the energy density is increased. The radius of gyration is reduced to compensate (first invariant, described above), but the path length must also be reduced. Thus the mirror points must be raised. Conversely, if the field weakens, mirror points can also be lowered and the particles could be lost into the atmosphere. The bounce period is only a weak function of the equatorial pitch angle of the particle (the angle between the particle velocity and the magnetic field line at the magnetic equator) since the particles spend most of their time at the mirror points. The bounce period can be approximated by [2]:

$$
\begin{aligned}
2 \pi / \Omega_{2} & =(4 \mathrm{~L} \mathrm{R} / \mathrm{v}) \mathrm{T}(\mathrm{y}) \\
\text { where: } & \mathrm{L}=\text { McIlwain's parameter } \\
& \mathrm{R}_{\mathrm{e}}=\text { Earth radius } \\
& \mathrm{y}=\sin \alpha \text { where } \alpha \text { is the equatorial pitch angle }
\end{aligned}
$$

and $T(y)$ is given by [2]:

$$
\mathrm{T}(\mathrm{y})=1.3802-.31985\left(\mathrm{y}+\mathrm{y}^{1 / 2}\right)
$$

\section{The Third Adiabatic Invariant}

Because the field has a radial gradient and curvature, the radius of curvature of the gyration or orbit about the field line is larger at the top of the orbit than at the bottom (top and bottom referenced with respect to the Earth radial direction). Thus the path does not quite close into a circle and the next orbit starts slightly eastward (for electrons) or slightly westward (for positive ions) from the previous gyration. This advance in position results in a drift motion around the Earth. After one drift period around the Earth, the particle will be back at the same location in the field where it started, provided the field is quiescent. The locus of points through which the particle passes is called its drift shell. The total flux enclosed by this shell must again be conserved. It is the conservation of this flux function, or third adiabatic invariant, that causes the particle drift shell to close after one drift period. However, during the time required for a particle to drift around the Earth (Figure 2), the magnetic field itself may change. During large magnetic storms, the change can be quite substantial, up to $1 \%$. After one drift period, the particle may find itself on a different drift shell with a different field intensity (and therefore a different energy). The resulting violation of the third adiabatic invariant is the primary source of particle acceleration in the magnetosphere.

The drift frequency is a function of the bounce frequency (note the T(y) in Eqn. 2 shows up in Eqn. 3) and can be approximately represented by [2]: 
$2 \pi / \Omega_{3}=-(3 \mathrm{~L} / 2 \pi \gamma)\left(\gamma^{2}-1\right)\left(\mathrm{c} / \mathrm{R}_{\mathrm{e}}\right)^{2}\left(\mathrm{~m}_{0} / \mathrm{qB}_{0}\right) \mathrm{D}(\mathrm{y}) / \mathrm{T}(\mathrm{y})$

where $\mathrm{D}(\mathrm{y})$ is given by

$$
D(y)=1 / 12\left\{5.5208-.4381 y-.6397\left(y \ln y+y^{1 / 2}\right)\right\}
$$

and $B_{0}$ is the value of the Earth's magnetic field at the surface of the earth at the equator. Figure 2 presents a summary of approximate gyration, bounce, and drift frequencies for electrons and protons as a function of energy and L.

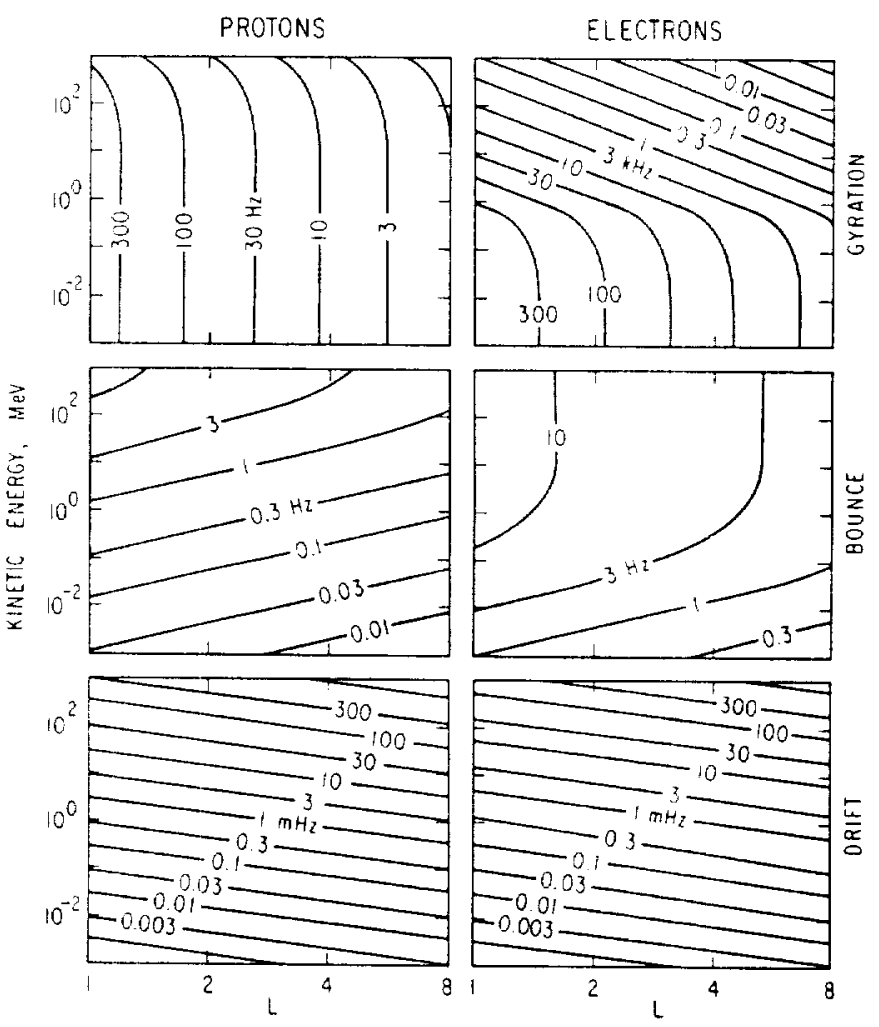

Figure 2. Gyration, Bounce and Drift Frequencies for Electrons and Protons in the Earth's Magnetic Field [2].

\section{McIlwain's L Parameter}

As a result of conservation of the first invariant, a particle's instantaneous pitch angle as it moves along a field line can be expressed in the form

$$
\operatorname{Sin}^{2} \alpha_{i} / B_{i}=\text { constant }
$$

where: $\alpha_{\mathrm{i}}$ is the particle pitch angle at location $\mathrm{i}$

$B_{i}$ is the magnetic field intensity at the same location

As a consequence of this relationship between $B$ and $\alpha$, if one knows the pitch angle of a particle at any point on the field line between the mirror points, one also knows $B_{m}$ (which is the point at which $\alpha=$ $90^{\circ}$ ). The loci of these mirror points as the particle drifts around the Earth are two rings of constant $B_{m}$ (one in each hemisphere). Provided one knows the unidirectional flux all along the field line below a point, the relationship expressed by the equation above permits the conversion of unidirectional fluxes to omnidirectional fluxes along the same region of the field line. In a similar manner, one can reconstruct the 
unidirectional flux from the omnidirectional flux. However, in practice it is much easier to measure the unidirectional flux along the field line (it can be done from a single equatorial point by using an instrument that scans in the angle $\alpha$ ) than to measure the omnidirectional flux distribution (which would have to be done by making measurements at closely-spaced points all along the field line).

As a particle drifts around the earth, the conservation of the second invariant results in the particle's guiding center tracing out a shell which connects the two rings of mirror points. The third invariant produces the result that the shell is closed upon itself--a particle remains on the same shell as it drifts around the earth. Of course, if the magnetic field varies during a drift period (or bounce or gyration), the adiabat associated with that motion will no longer be precisely conserved. As previously stated, such violation of conservation due to magnetic field fluctuations results in pitch angle diffusion, cross-field particle diffusion, and in changes in the energy of the particles.

Mapping of the particle population in the magnetosphere requires multi-dimensional labelling: particle species; energy; pitch-angle; altitude, latitude, longitude. The task of mapping the radiation environment is greatly simplified by reducing the three spatial coordinates to two magnetic coordinates, B and L, which are essentially the drift shells $(\mathrm{L})$ and mirror rings $\left(\mathrm{B}_{\mathrm{m}}\right)$ described above. The adiabatic invariant associated with the bounce motion, $I$, is obtained by integrating the function $\left(1-B / B_{m}\right) 1 / 2$ between the mirror points. Since this is awkward to do in a nonuniform field (the field has to be represented by a multipole expansion), an approximate relation is derived which can be related to a dipole field: $\mathrm{L}^{3} \mathrm{~B} / \mathrm{M}=\mathrm{F}\left(\mathrm{I}^{3} \mathrm{~B} / \mathrm{M}\right)$. Here $M$ is the dipole moment of the geomagnetic field. The function $F$ can be calculated at a number of points, generating an interpolation table. L then becomes a simple calculation [1]. Note that for the real magnetic field, L is only an approximate representation of I, although a sufficiently accurate representation for mapping purposes. For a dipole, $L=R$, where $R$ is the normalized distance from the center of the dipole to the equatorial crossing of the field line labeled "L". For our purposes, the dipole approximation will provide some understanding without belaboring the mathematics:

$$
\begin{aligned}
& R=L \cos ^{2} \lambda \\
& B=M / R^{3}(4-3 R / L) 1 / 2
\end{aligned}
$$

where $\mathrm{R}$ and $\lambda$ are the usual radial distance and magnetic latitude in a dipole field, $\mathrm{M}$ is the dipole moment, and $L$ is McIlwain's parameter. Note that $R$ and $\lambda$ are not sufficient to describe the spatial characteristics of the particle distributions since a given particle does not drift at a constant $R$ or mirror at a constant $\lambda$ except in a true dipole field where the azimuthal symmetry produces a degeneracy. The above expression shows that in a dipole field, $L$ would correspond to the radial distance from the center of the Earth to the equatorial crossing of the magnetic field $\left(\lambda=0^{\circ}\right)$.

\section{The Radiation Belts}

The Earth's magnetosphere contains a wide variety of charged particles, primarily electrons and protons, with energies ranging from the thermal (less than $1 \mathrm{eV}$ ) to highly relativistic (tens of MeV for electrons, $\mathrm{BeV}$ for protons). The ionosphere contains a cold plasma, in the $1 \mathrm{eV}$ energy range, with densities of the order of $106 / \mathrm{cm}^{3}$. The ionosphere is generally considered to consist of the neutral and ion components up to about $1000 \mathrm{~km}$ altitude, with the region above this called the plasmasphere (since the constituents there are highly ionized, forming a plasma). The plasmasphere particle density drops slowly until a boundary, called the plasmapause, is reached in which the cold plasma density drops abruptly by about 2 orders of magnitude, from $10^{3}$ to $10^{4}$ per $\mathrm{cm}^{3}$ to below 100 per $\mathrm{cm}^{3}$. The location of the plasmapause is local-time and magnetic-activity dependent but is generally found between $\mathrm{L}=4$ and $\mathrm{L}=5$ and generally follows the field line to higher latitudes rather than an altitude contour. Beyond this region, hot plasma clouds, with temperatures of the order of 1 to $10 \mathrm{keV}$ or more, are sometimes encountered. The plasma is heated by magnetic processes in the tail and auroral regions of the magnetosphere. In the remainder of this section, we will restrict our discussion to the energetic particle populations $>40 \mathrm{keV}$. They will be discussed by location or zone and by species. 
When Prof. Van Allen's detector passed through the magnetosphere in a radial direction, the count rate in the detector first increased, then decreased, and then increased again. Two distinct zones of trapped radiation were being traversed. These were named the Van Allen belts and are commonly referred to as the inner zone and the outer zone, with a region known as the slot separating them. Only for electrons are these zones distinct. Figure 3, which presents data from a period when copious fluxes of fission electrons were still present from the Starfish nuclear explosion in space, shows the minimum between the inner zone and outer zone. The inner zone, which is generally considered to cover the region $1.0<\mathrm{L}<2$, has a peak in flux intensity at about $\mathrm{L}=1.5$ for $1 \mathrm{MeV}$ electrons. The region $2.0<\mathrm{L}<2.8$ is generally considered the slot region where magnetospheric processes result in a low intensity of electrons during magnetically quiet periods. The process which removes the electrons is a resonant interaction between energetic electrons and whistler-mode (right circularly polarized) electromagnetic waves. The interaction results in some of the particles being scattered to lower angles (relative to the magnetic field line) such that their new mirror points are within the atmosphere. The atmosphere absorbs them. Thus the slot region normally contains relatively low fluxes of particles. At times of large magnetic storms, the slot can be refilled and quite high flux levels can be observed there for a few days. The location of the slot is quite variable: during large geomagnetic storms, the minimum between the inner and outer zone can be very narrow and may be displaced to a low $L$ value, even centered as low as $L=2.0$. Immediately after a storm, the slot may be completely filled with electrons and so does not exist. An extensive discussion of these dynamics is available elsewhere [3]. The outer electron zone, which is extremely variable, typically peaks around $3.5<\mathrm{L}<4.0$. In the outer zone, significant fluxes of electrons with energies in excess of $5 \mathrm{MeV}$ are observed after major magnetic storms. During extended quiet periods, the outer zone may almost disappear at high energies. The difference in flux intensity from minimum to maximum may be as high as 5 orders of magnitude. See, for example, Figure 2 of [3] or Figure 7 of [4].

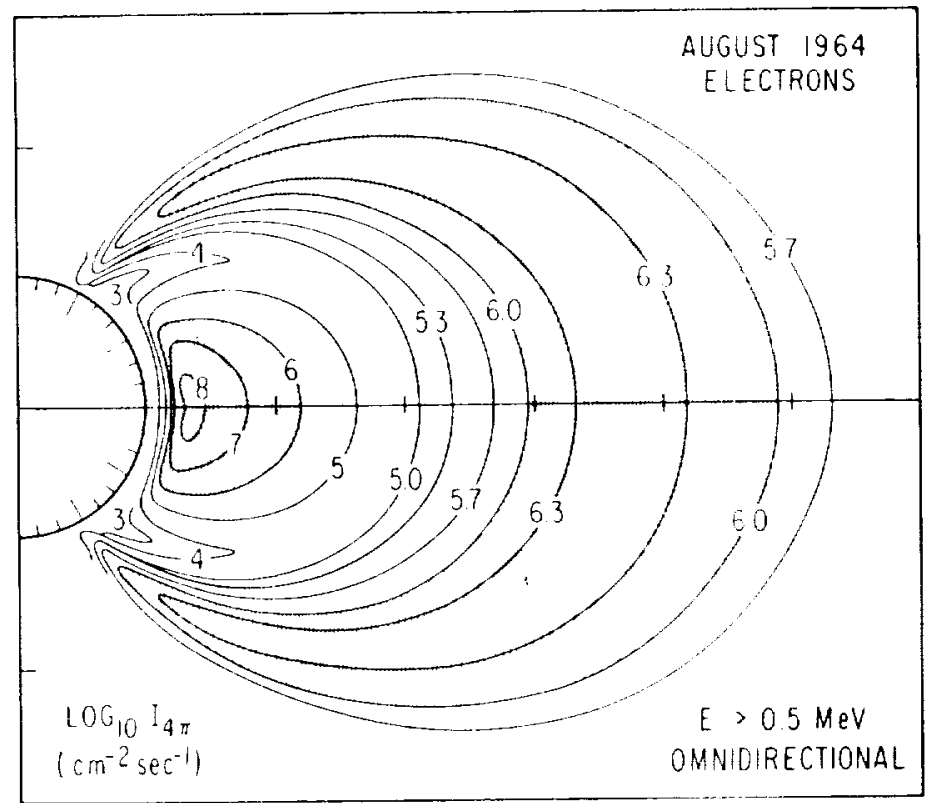

Figure 3. Inner and Outer Zone Electron Belts. The numbers on the contours are the $\log _{10}$ of the integral omnidirectional flux, $\mathrm{I}_{4 \pi}$. The intense inner zone was due to the Starfish fission source. For times subsequent to 1968 , the maximum inner zone intensity is several orders of magnitude lower.

The proton environment is sometimes separated into two constituents also, but in this case the separation is done on the basis of the energy of the protons. The same region of space that constitutes the inner zone for electrons contains very energetic protons, some with energies in excess of $200 \mathrm{MeV}$, which are also the result of the cosmic-ray albedo neutron decay described in the section on inner zone electrons.

There is another source of trapped protons. Low energy protons, from either a solar wind or ionosphere source, are accelerated similarly to the energetic electrons. Some of the initial acceleration for 
ionospheric electrons is produced by electric fields, especially in the auroral zone. The low energy proton belt can be considered to be composed primarily of protons with energies below $10 \mathrm{MeV}$. These lowerenergy protons are present in both the inner and outer zones. There is no slot such as occurs for the electrons. The peak in proton flux intensity depends on the energy, with lower energy protons peaking farther out. The $1 \mathrm{MeV}$ proton flux is at its maximum at about $\mathrm{L}=3$.

\section{TRAPPED PARTICLE POPULATIONS}

In this section, we will briefly describe the four major components of the magnetospherically trapped energetic particle population---the inner and outer electron zones, the energetic proton belt, and the low energy proton population. The sources of the particles and their general flux levels as a function of $L$ and energy will be discussed. Solar flare protons, which are discussed in the later section on modelling, will not be discussed in this section because the contribution of flare protons which become trapped to the fluences observed in most orbits is negligible. Flare protons in the polar region may be a major concern for some satellites, but those particles are not trapped.

\section{Inner Zone Electrons}

The source of the inner zone electrons is a combination of cosmic-ray albedo neutron decay (CRAND) and radial diffusion through the slot from the outer zone. In the CRAND mechanism, cosmic rays interact with air molecules in the upper atmosphere, producing energetic neutrons, some of which escape back into space. Since neutrons are uncharged, they cross magnetic field lines unimpeded. However, some decay while still in the magnetosphere and the decay products, an electron and a proton, are charged and so become trapped. The end-point energy of the electron in neutron decay is slightly under $1 \mathrm{MeV}$. The contribution of the neutron's velocity to the electron's energy is small. As a result, there are few electrons with energies in excess of $1 \mathrm{MeV}$ in the inner zone. Electrons with higher energies are present in small numbers, especially above about $L=1.65$ after large magnetic storms, but can be ignored as a hazard to space systems except for their background effects in sensors.

Inner zone electrons below about $1000 \mathrm{~km}$ have lifetimes that are primarily determined by the scale height of the atmosphere. During solar-active periods, the increased scale height results in a reduced lifetime and lower average fluxes. This is reflected in the models by having a solar maximum and a solar minimum version. Farther out in the inner zone, electrons are quite stable, with typical lifetimes of 400 days [5]. Principle loss mechanisms are probably any or all of the following: radial diffusion into the atmosphere (violation of the second and third invariants caused by magnetic storms); interaction with whistler-mode waves produced by lightning strokes (the resonant interaction between these waves, also known as cyclotron waves, and the electrons results in a lowering of the electron pitch angle, causing it to be absorbed by the atmosphere at the end of the field line); interaction with VLF waves from ground-based transmitters.

The order-of-magnitude of the electron fluxes at $\mathrm{L}=1.45$ in the inner zone are as follows: $>10^{8}$ for $\mathrm{E}_{\mathrm{e}}$ $>0.1 \mathrm{MeV} ;>106$ for $\mathrm{E}_{\mathrm{e}}>1 \mathrm{MeV} ;>105$ for $\mathrm{E}_{\mathrm{e}}>2 \mathrm{MeV}$. The numbers represent the integral, omnidirectional fluxes $\mathrm{cm}^{-2}-\mathrm{sec}^{-1}$. Below about $\mathrm{L}=1.55$, the fluxes are quite stable, with little variation being observed over the solar cycle [6] except for altitudes below $1000 \mathrm{~km}$ where atmospheric effects are observed. Above $\mathrm{L}=1.6$, major magnetic storms inject electrons with energies up to at least $1.2 \mathrm{MeV}$ [3]. Figure 4 shows the equatorial omnidirectional inner zone electron flux intensities as a function of $L$ and energy.

\section{Outer Zone Electrons}

The outer zone electrons originate either as solar wind electrons in the tail of the magnetosphere or as ionospheric electrons at high latitudes which are accelerated up the field lines. Magnetic field fluctuations cause them to be diffused radially inward, energizing them. The acceleration is a consequence of the conservation of the first adiabatic invariant coupled with violation of the third invariant, discussed earlier, by magnetic activity. As the particles are transported to field lines deeper in the magnetosphere, the increase in the average field intensity has to be compensated by an equivalent increase in particle momentum, or energy. The various fluxes peak at different locations in the outer zone for different energies, with the higher energies peaking at lower L. Representative outer zone fluxes are of the order of: $>10^{8}$ for $E_{e}>$ 
$0.1 \mathrm{MeV}, \mathrm{L}=6 ;>10^{7}$ for $\mathrm{E}_{\mathrm{e}}>1 \mathrm{MeV}, \mathrm{L}=5 ;>105$ for $\mathrm{E}_{\mathrm{e}}>4 \mathrm{MeV}, \mathrm{L}=4$. Units are as described for the inner zone fluxes.

Outer zone electron fluxes are highly variable, with increases at a given energy on a given $\mathrm{L}$ shell being as great as 5 orders of magnitude in less than a day. These large increases are caused by major magnetic storms, where $D_{s t}>-150 \gamma . D_{s t}$ is a global magnetic field disturbance index which is generally responsive to low latitude variations caused by a magnetospheric ring current. This ring current is composed of low energy ions accelerated by the magnetic storm. Typical decay constants for outer zone electrons are of the order of 10 days. In addition to the radial diffusion of particles caused by magnetic storms, they also cause pitch-angle scattering of the particles. Thus particles which were previously stably trapped on a field line (had mirror points that were above the atmosphere everywhere along their drift paths), can be perturbed so that they now mirror within the residual atmosphere below $100 \mathrm{~km}$ at some point along their drift path. At this altitude, the atmosphere absorbs the particles. A low altitude satellite which is normally below the trapped radiation zones (except when traversing the South Atlantic Anomaly) may suddenly find itself bathed in large fluxes of energetic electrons at midlatitudes when it encounters these particles which show up low on the outer zone field lines (sometimes called the horns of the outer zone). The South Atlantic Anomaly is a region of anomalously low magnetic field strength. Since particles mirror at a constant $B_{m}$, they attain at their lowest mirror altitude in the South Atlantic Anomaly.

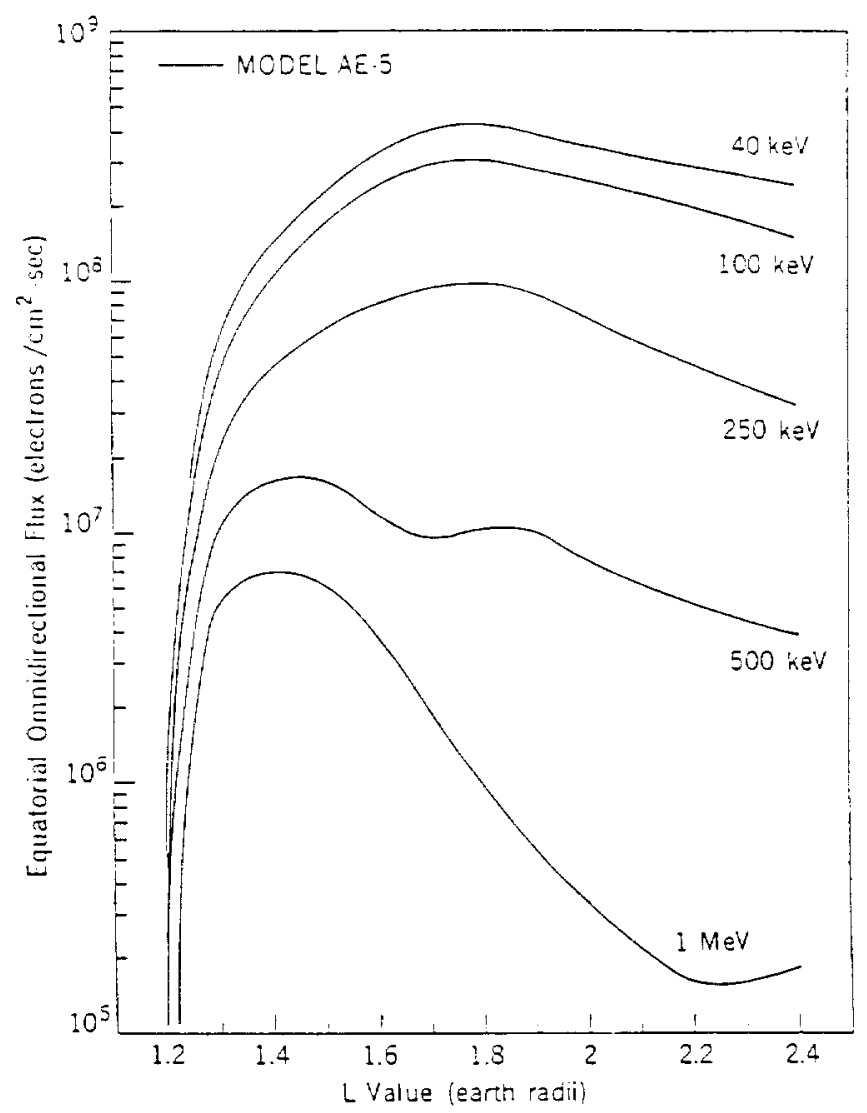

Figure 4. Inner Zone Electron Flux Intensities [6]

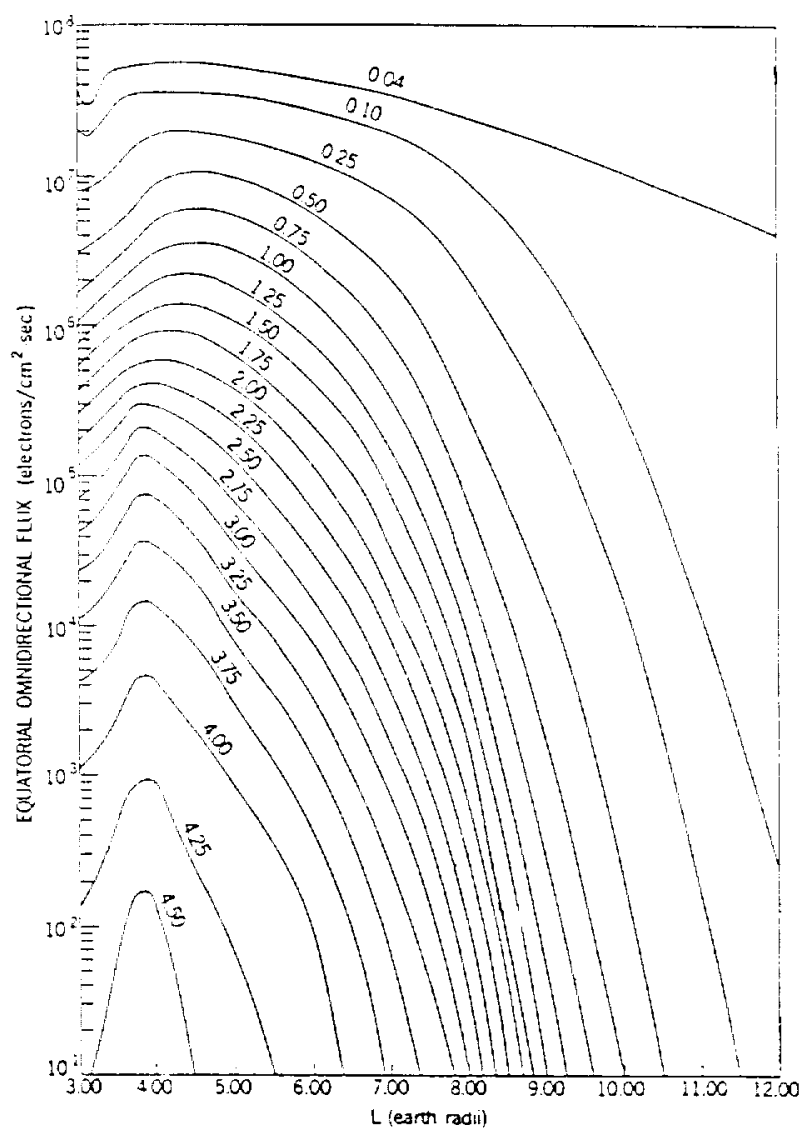

Figure 5. Outer Zone Electron Flux Intensities [7]

\section{Energetic Protons}

The source of the energetic protons which are present in the inner zone is CRAND, the mechanism mentioned previously. They are quite stable, with minor variations in intensity occurring at low altitudes due to variations in the atmospheric density due to solar activity. Typical intensities are of the order of 
$>10^{4}$ for $E_{p}>100 \mathrm{MeV}$ and $>103$ for $E_{p}>300 \mathrm{MeV}$, both at $\mathrm{L}=1.45$. Due to the secular variation in the magnetic field, a very slow decrease which may be an indication that the earth's field will undergo a reversal in the geologically-speaking near future (104 years?), the energetic proton environment is also exhibiting a small decrease (the decreasing field intensity is driving the protons into the atmosphere, again due to conservation of the adiabatic invariants). A serious problem in particle modelling due to this secular decrease in field intensity will be discussed in the section on models.

APBMIN OMNIDIRECTIONAL FLUX (PROTONS/CM²-SEC)

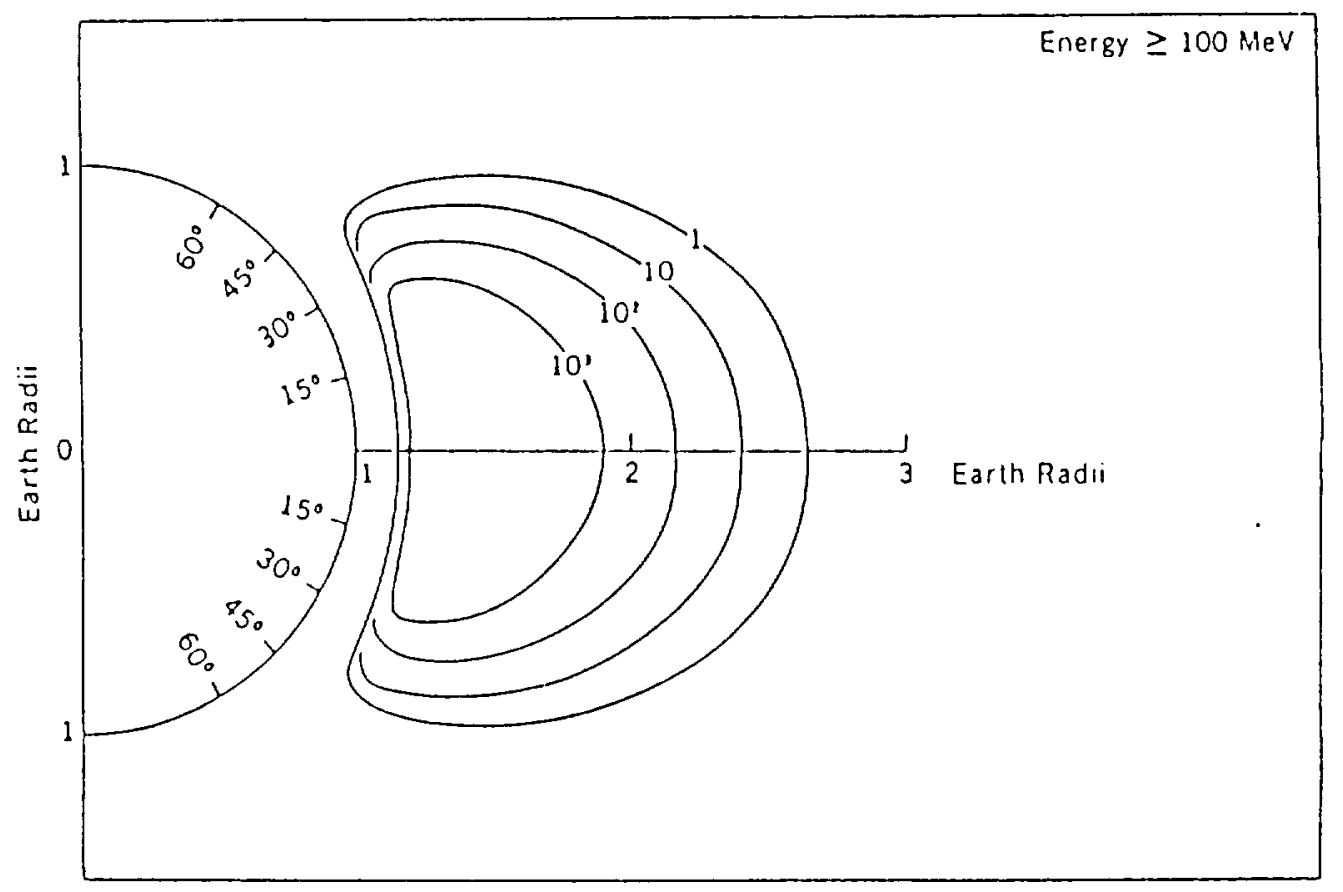

Figure 6. High Energy Protons in the Inner Zone [8]

\section{Low Energy Protons}

The low energy protons with which we are concerned here are in the 0.5 to $5 \mathrm{MeV}$ range, since there are large fluxes of such particles in both the inner and outer zone and they have significant materials effects. Particles with these energies can originate in a number of sources: radial diffusion and energization of solar wind particles which enter the geomagnetic tail, similar to the outer zone electrons; ionospheric acceleration up field lines, with subsequent radial diffusion and acceleration; direct access of solar flare protons. Typical intensities in the outer zone are $>10^{8}$ for $\mathrm{E}_{\mathrm{p}}>0.1 \mathrm{MeV} ;>10^{7}$ for $\mathrm{E}_{\mathrm{p}}>1 \mathrm{MeV} ;>105$ for $E_{p}>10 \mathrm{MeV} ;<102$ for $E_{p}>100 \mathrm{MeV}$. Again, these are omnidirectional, integral fluxes in units of $\mathrm{cm}^{-2}$-sec-1. While the fluxes are subject to variation due to magnetic storm activity, the variations are much smaller than for electrons. The primary loss mechanisms are deenergization through collisions with the residual atmosphere and charge-exchange, which results in an energetic neutral particle which is not trapped by the magnetic field.

\section{CURRENT STATUS OF PARTICLE MODELS}

In this section, we will cover the currently recommended particle models, their ranges and estimates of accuracy, discuss briefly their sources, availability, future modelling plans, and requirements for additional data. A more extensive discussion of the modelling efforts at the National Space Science Data Center is presented elsewhere [9]. 


\section{Inner Zone Electron Models}

The current NSSDC models which provide useful inner zone $(\mathrm{L}=1.2$ to 2.4$)$ electron data are AE5 [6], for solar minimum, AE6 [10], for solar maximum, and AE8 [9] for either solar minimum or maximum. The energy range of these models is from $0.04 \mathrm{MeV}$ to $5 \mathrm{MeV}$, although present techniques can not make reliable measurements of electrons with energies above $2 \mathrm{MeV}$ below about $\mathrm{L}=1.55$ in the inner zone. They are empirical models, being based on in-situ measurements of the fluxes. To produce the models, data is acquired from investigators, corrected, edited, averaged, interpolated, extrapolated, etc; in other words, it is all very thoroughly massaged. Some estimate of its reliability is also made, but such a step is very subjective. In general, the temporal coverage for any data set is six months or longer. The measurements were made between 1964 and 1977 (although data up to 1968 include Starfish electrons). Starfish contamination has been removed from the data in generating the above models. Newer data are available for incorporation into models [9]. The accuracy of the models is very good, better than a factor of two, for energies below $1 \mathrm{MeV}$ and $\mathrm{L}<1.65$. Above $\mathrm{L}=1.65$, the variability of the flux levels themselves produce uncertainty. Above $2 \mathrm{MeV}$, the fluxes are extrapolations and their accuracy is unknown.

The models, and codes for running them, are available from NSSDC. In addition to a tape format, the codes are also available as files on a VAX at NSSDC. The VAX is a SPAN (Space Physics Analysis Network) node. Thus, anyone with access to SPAN or other networks which can connect to SPAN (such as TELENET, ARPANET, BITNET, etc.) can access these files to download them to their home computer via the network. Dr. James Green is in charge of SPAN at NSSDC. Alternatively, the codes can also be run interactively on the NSSDC VAX at no charge to the user (other than his own costs of connecting to the SPAN network). An alternate interactive resource is EnviroNET [11], which is resident on a MicroVAX at GSFC and is accessible as the SPAN node ENVNET. To access EnviroNET, the user name ENVIRONET and password HENNIKER are used.

Future plans for the inner zone modelling activity are to incorporate newer data bases and also perhaps include some storm-time dynamics. It may not be possible to accomplish the latter task with presently available data bases, but the CRRES mission [12] has as one of its objectives the acquisition of the data required for producing dynamic particle models.

\section{Outer Zone Electron Models}

In-situ electron flux data in the outer zone are far from satisfactory for generating electron models. Most of the data used are extrapolations in both energy and altitude. At the geosynchronous region, measurements up to $1.7 \mathrm{MeV}$ have been available from ATS-1. Near the equator, $\mathrm{S}^{3}$ provided measurements from about $\mathrm{L}=2.5$ to $\mathrm{L}=6$, but only up to $300 \mathrm{keV}$. OGO- 5 had an electron channel at $2.7 \mathrm{MeV}$ but the satellite orbit inclination was $27^{\circ}$ so it made no measurements near the equatorial region. All other satellites which traversed the equatorial region at high altitude either had no high energy electron measurements $\left(E_{e}>1.5 \mathrm{MeV}\right)$ or the energy threshold and detector efficiency were not known with sufficient accuracy to be usable in modelling. The source of the orders-of-magnitude discrepancies seen in comparisons of energetic outer zone electrons (e.g., Figure 12 of [4]) are these uncertainties. All other sources of data used in the models are extrapolations of measurements made low on the field line.

The current NSSDC outer zone models $(L>2.4)$ which provide useful results are the following: AE7Lo and AE8 for solar minimum and AE7-Hi for solar maximum or long duration missions ( $>5$ years). For geosynchronous satellites, another model is still relatively valid--AE3 [13]. For long term missions, AE7$\mathrm{Hi}$ is probably accurate to within a factor of two, especially for $\mathrm{L}<6$ and $\mathrm{E}<5 \mathrm{MeV}$. However, if the mission includes the period a year or two following the sunspot maximum when the magnetic storm activity is greatest, AE7-Hi will err on the low side (actual integrated fluences can be expected to be greater than the model prediction). AE7-Hi was generated in response to criticisms that the earlier models, AE4 and AE6, were deficient in high energy electrons, and seriously so. In fact, in those models the energy spectra were truncated at $5 \mathrm{MeV}$, as is also done in $\mathrm{AE} 8$. A comparison of the models with in-situ data [4] showed that the models predicted fluxes that were low by about a factor of three, but almost the entire deficiency was in electrons $>1.5 \mathrm{MeV}$. The result was a prediction of dose in heavily shielded satellite components that was low by an order of magnitude. AE7- $\mathrm{Hi}$, which truncates the energy spectrum at $7.5 \mathrm{MeV}$, has also been criticized for truncating the spectrum, since electrons with energies up to $10 \mathrm{MeV}$ have been 
measured at geosynchronous orbit [14]. Figure 7 shows the equatorial flux contours as a function of $L$ and energy which are contained in AE7-Hi [15].

AE8 exists in two forms, AE8MIN and AE8MAX, which are supposed to represent the environment during solar minimum and maximum. However, both are truncated at $5 \mathrm{MeV}$ and cannot properly model the solar maximum period when large fluxes of very energetic electrons appear. AE8 uses a single outer zone model and uses AE5 and AE6 solar minimum and solar maximum inner zone models. The major difference between AE8 and AE7-Hi is in the high energy electron flux at around L = 4. The AE7-Hi model contains about a factor of two greater flux at $3 \mathrm{MeV}$ and about a factor of 10 greater flux at $4.5 \mathrm{MeV}$. At solar minimum, there are relatively few energetic electrons and any of the later models, AE7-Hi, AE7-Lo, AE8MIN, and AE8MAX are satisfactory for any use except calculating background rates in detectors. The models are probably accurate to a factor of three for dose calculations.

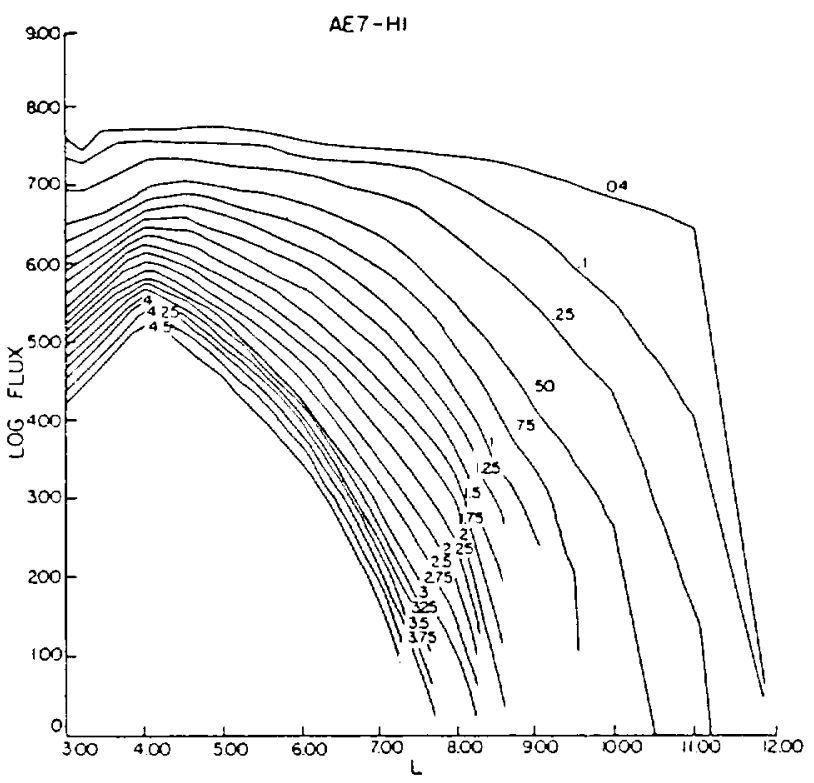

Figure 7. AE7-Hi Equatorial Electron Flux Contours [15].

As in the inner zone case, future plans are to incorporate new data in an attempt to increase the accuracy of the models and to include a dynamic model (addressing both the prediction of electron increases due to magnetic storms and the evolution of the fluxes after the increases). The Radsat portion of the CRRES mission [12] has two outer zone electron modelling goals: obtain data in the outer zone nearer to the equator than has been done previously in order to reduce the amount of extrapolation that has been necessary for model generation; obtain a data base which can be used to generate a dynamic model.

\section{Proton Models}

The current proton models are AP8MIN and AP8MAX, again representing the solar minimum and maximum periods. The major effect of the solar variation is the variation in atmospheric density at lower altitudes: at solar maximum, the higher scale height of the atmosphere decreases the energetic proton fluxes. The models are probably accurate to $50 \%$ or better. They cover the energy range from $100 \mathrm{keV}$ to $400 \mathrm{MeV}$ and the L range from 1.17 to 7 . The data were obtained during the same time period that the inner zone electron data was obtained. Figure 8 shows the equatorial flux contours as a function of $L$ and energy provided by AP8MIN. Since the MIN model predicts slightly more flux than the MAX model, it can be used during solar maximum or for long term missions as a conservative model.

One major problem with the energetic proton models is the fact that they are organized in terms of $\mathrm{B}, \mathrm{L}$. The secular variation in the earth's magnetic field (the dipole term is diminishing) causes the energetic proton ensemble, which is nominally very stable, to be carried to lower altitude. The model does not take into account the increased atmospheric density the protons will encounter at the lower altitude. As a result, if calculations are made with the magnetic field projected well into the future (more than ten to fif- 
teen years), totally invalid results are obtained for low altitudes such as the Space Station orbit [16]. Since the source of the energetic protons is the decay of energetic neutrons produced in the upper atmosphere by cosmic rays, the geometry of the production process relative to the atmosphere will not change. The future configuration of the inner zone proton belt probably will not change relative to the present configuration, provided both are described in terms of $L$ and $B / B_{0}$, where $B$ is the magnetic field at the point in question and $B_{o}$ is the equatorial intensity on the same field line. One will almost certainly get a more accurate result for a calculation of the proton environment for Space Station in the year 2025 by making the calculation with the present field model than by extrapolating the field 35 years into the future.

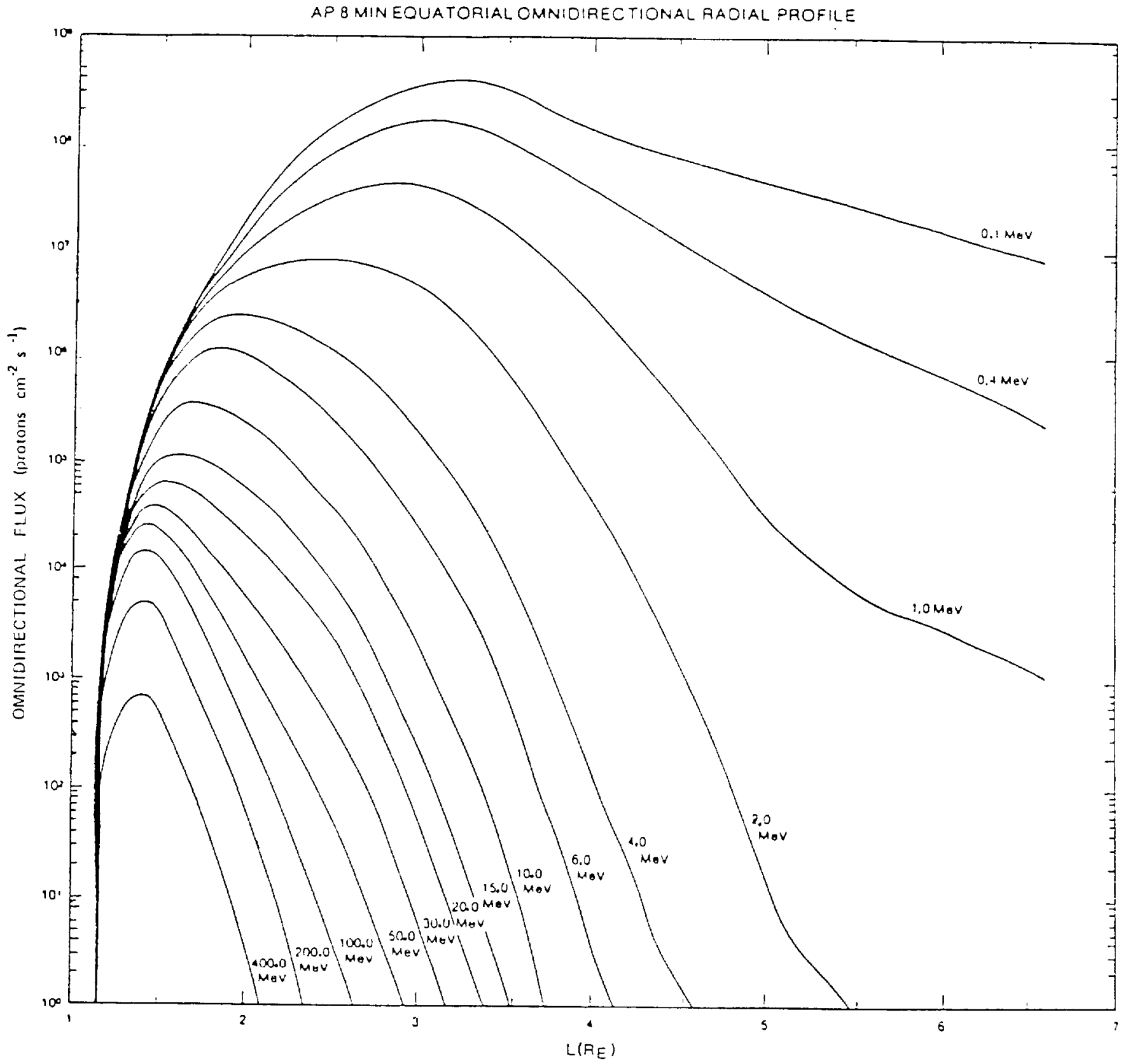

Figure 8. AP8MIN Equatorial Proton Flux Contours [15]. 


\section{Solar Flare Proton Models}

Solar flare protons are treated separately because they are transient and show up in the outer zone only in relatively small numbers. Their major impact is in the case of low altitude polar-orbiting satellites which normally receive relatively little particle irradiation--and almost all of that in short infrequent traversals of the South Atlantic Anomaly region. For some satellites, the major portion of the particle environment they encounter during their lifetime may occur during one or two major solar flare particle events, each lasting only a few days. In a typical solar cycle of 11 years, $90 \%$ of the energetic proton fluence is the result of a single anomalously large flare. The integrated fluence over the polar caps from the one event can be of the order of $2 \times 10 \% / \mathrm{cm}^{2} \mathrm{P}+>30 \mathrm{MeV}$. Averaged over a solar cycle, the annual fluence is about $5 \times 108 / \mathrm{cm}^{2} \mathrm{P}+>30 \mathrm{MeV}$ over the polar cap. At $100 \mathrm{MeV}$, these numbers are about a factor of 30 lower. A low altitude polar orbiting satellite spends roughly $40 \%$ of its time at latitudes where these protons can gain access. Thus such a satellite may receive virtually all of its energetic proton dose from solar flares rather than from the magnetospherically-trapped protons.

\section{FUTURE MODELLING REQUIREMENTS}

The major efforts in modelling must be directed toward the electron environments, since the energetic inner zone protons are relatively stable and well understood and the outer zone protons can be described well statistically. Within limits, their evolution after a storm is also generally predictable. This is primarily due to the relative ease with which proton measurements can be made. Also, the studies of the physics of the protons have been more productive. The CRRES mission [12] is expected to provide additional high-quality data for the proton dynamics modelling.

While a large amount of data is available which has not yet been used to update the various trapped radiation models, there are significant gaps in the electron data bases. A major reason for this is the difficulty of separating the signal from a small flux of these particles from the large background of protons and cosmic rays. One of these gaps was mentioned previously: No useful data base has ever been obtained in the outer zone for electrons above $300 \mathrm{keV}$ between about $\mathrm{L}=2.8$ and geosynchronous orbit at geomagnetic latitudes below about $20^{\circ}$. CRRES will lower this limit to $10^{\circ}$. Above $2.8 \mathrm{MeV}$ no data is available for altitudes above $8000 \mathrm{~km}$ except for some geosynchronous orbit measurements [14]. In the inner zone above $2 \mathrm{MeV}$, no usable measurements are available because of tremendous penetrating proton background problems in detectors. CRRES will not be able to furnish this type of data, either, because its primary radiation mission is an engineering one and electrons above $2 \mathrm{MeV}$ in the inner zone can be ignored. (If you have problems measuring them, they are unlikely to cause a problem on operational vehicles.)

One major area which has not been modelled is the dynamics of the radiation belts in response to magnetic activity. One report [17] attempts to correlate the response of the outer zone to the magnetic index $\mathrm{K}_{\mathrm{p}}$, but the particle data were all obtained at geosynchronous orbit and the field lines that are represented in the $\mathrm{K}_{\mathrm{p}}$ index are high latitude field lines which thread through the geosynchronous region. Hence, that study is limited in validity to the geosynchronous region. Low altitude measurements indicate that in general the outer zone does not correlate with $\mathrm{K}_{\mathrm{p}}$, except incidentally when major magnetic storms occur, but does have some relationship with $\mathrm{D}_{\mathrm{st}}$, the low latitude index mentioned previously. The intention is to get the type of data base from CRRES that one needs to address the dynamics modelling.

In order to be useful, the dynamics models have to address three issues quantitatively: a) The prediction of magnetic storms which produce increases in the outer zone energetic electron and proton populations; b) The energy spectra and L profiles of the fluxes as a function of the magnetic storm parameters; and c) The evolution of the distribution as a function of time (and magnetic activity) post-storm. The first of these requires a better understanding of the solar-terrestrial coupling physics and may have to await completion of the Global Geophysics Program (aka International Solar-Terrestrial Physics program) which is scheduled to start launching satellites in the 1993 time period [12]. A first step for b) and c) would be to make a statistical model of particle storm and post-storm behavior from a large data base. Again, CRRES is designed to provide the data base for such an effort and current plans include developing such a statistical model. 


\section{REFERENCES}

[1] McIlwain, Carl E., Coordinates for Mapping the Distribution of Magnetically Trapped Particles, $J$. Geophys. Res., 66, 3681-3691, 1961.

[2] Schulz, M. and L. J. Lanzerotti, Particle Diffusion in the Radiation Belts, Springer, New York, 1974.

[3] Vampola, A. L., "Natural Variations in the Geomagnetically Trapped Electron Population," in Proceedings of the National Symposium on Natural and Manmade Radiation in Space, NASA TM X2440, E. A. Warman, Ed., 1972.

[4] Vampola, A. L., J. B. Blake and G. A. Paulikas, "A New Study of the Magnetospheric Electron Environment," J. Spacecraft and Rockets, 14, 690, 1977.

[5] Stassinopoulos, E. G. and P. Verzariu, General formula for decay lifetimes of Starfish electrons, $J$. Geophys. Res., 76, 1841, 1971.

[6] Teague, M. J., and J. I. Vette, The Inner Zone Electron Model AE-5, NSSDC WDC-A-R\&S 72-10, 1972.

[7] Singley, G. W., and J. I. Vette, A Model Environment for Outer Zone Electron, NSSDC WDC-A$R \& S 72-13,1972$.

[8] Sawyer, D. M., and J. I. Vette, AP-8 Trapped Proton Environment, NSSDC WDC-A-R\&S 76-06, 1976.

[9] Bilitza, D., D. M. Sawyer, and J. H. King, Trapped Particle Models at NSSDC/WDC-A-R\&S, NASA CP-3035, p. 569, May 1989.

[10] Teague, M. J., K. W. Chan and J. I. Vette, AE-6: A Model Environment of Trapped Electrons for Solar Maximum, NSSDC WDC-A-R\&S 76-04, 1976.

[11] Lauriente, M., EnviroNET: Space Environments for SDIO Experiments, this proceedings.

[12] Hardy, D. A.. The Radiation Belt Mission on CRRES, Presented at the Space Environmental Effects on Materials Workshop, June 1988.

[13] Vette, J. I. and A. B. Lucero, Models of the Trapped Radiation Environment, Vol. III: Electrons at Synchronous Altitudes, NASA SP-3024, 1967.

[14] Baker, D. N., R. D. Belian, P. R. Higbie, R. W. Klebesadel, and J. B. Blake, "Hostile Energetic Particle Radiation Environments in Earth's Outer Magnetosphere," in The Aerospace Environment at High Altitudes and its Implications for Spacecraft Charging and Communications, AGARD CP 406, p. 4-1, 1986.

[15] Handbook of Geophysics and the Space Environment, A. S. Jursa, Ed., AFGL/AFSC, 1985. NTIS Accession No. ADA 167000.

[16] Konradi, A. and A. C. Hardy, "Radiation Environment Models and the Atmospheric Cutoff" $J$. Spacecraft and Rockets, 24, p. 284, 1987.

[17] Nagai, T, "Space weather forecast: Prediction of relativistic electron intensity at synchronous orbit," Geophys. Res. Lett., 15, 425,1988. 